\title{
Correction: Hypothermic oxygenated perfusion inhibits HECTD3-mediated TRAF3 polyubiquitination to alleviate DCD liver ischemia- reperfusion injury
}

Wei Zhou (D, Zibiao Zhong, Danni Lin, Zhongzhong Liu, Qiuyan Zhang 10, Haoyang Xia, Sheng Peng, Anxiong Liu, Zhongshan Lu, Yanfeng Wang, Shaojun Ye (i) and Qifa Ye (1)

Correction to: Cell Death and Disease https://doi.org/10.1038/s41419-021-03493-2

published online 24 February 2021
The original version of this article unfortunately contained an error in Figure 4. The H\&E stained picture in the HOPE group in Figure 4 was wrong. The authors apologize for the error. The correct Figure 4 can be found 
A

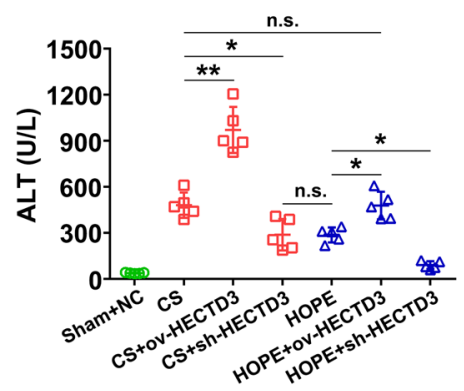

C
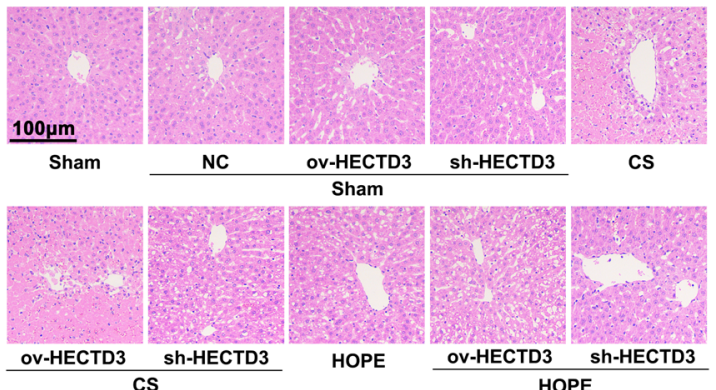

E

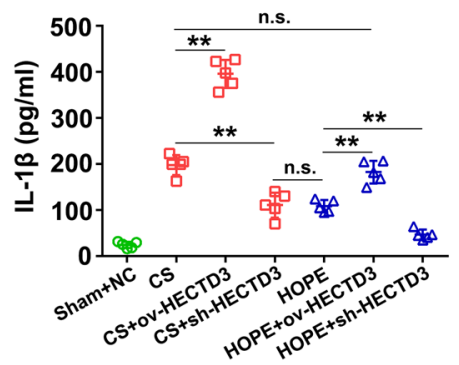

B

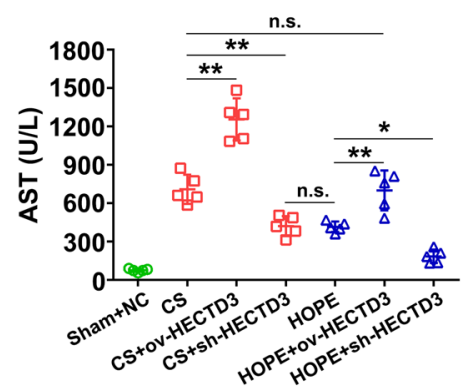

D

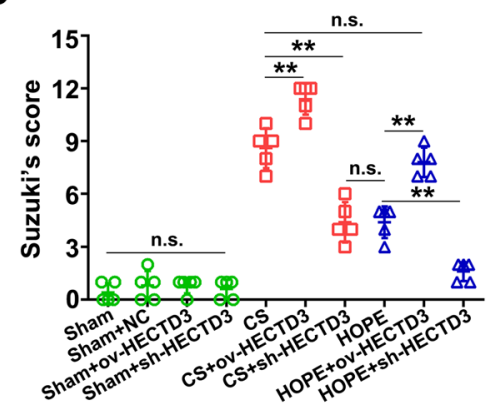

F

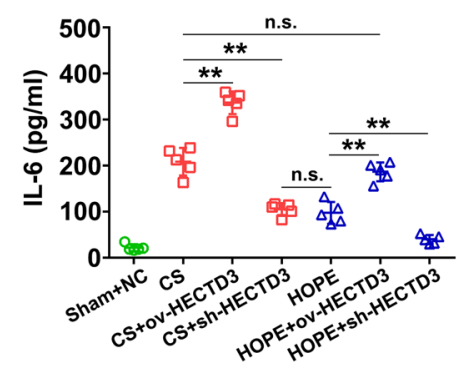

G

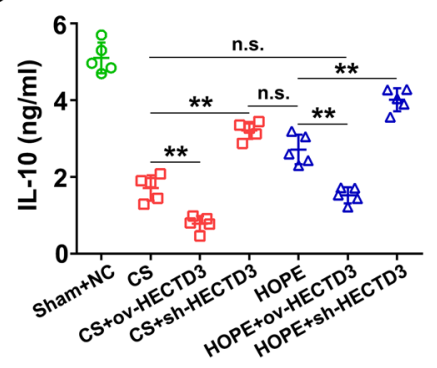

